\title{
Novel incretin receptor antibodies combat weight gain
}

\section{cef}

Strikingly, the combination of muGIPR-Ab and liraglutide led to a $23.5 \%$ decrease in weight

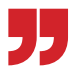

Agonists of the receptor for the gut-derived incretin hormone, glucagon-like peptide 1 (GLP1), have proved efficacious for the treatment of obesity and type 2 diabetes owing to their ability to promote satiety and postprandial insulin secretion. Writing in Science Translational Medicine, Lloyd and colleagues now report an alternative approach to intervening in incretin signaling - antibodies targeting the receptor of another gut-derived incretin hormone, glucose-dependent insulinotropic polypeptide (GIP) - that prevents weight gain and reduces food intake in mouse and monkey models of obesity.

GIP, which is secreted from $\mathrm{K}$ cells in the proximal small intestine in response to dietary carbohydrate and fat, acts on the GIP receptor (GIPR) in pancreatic $\beta$-cells and adipocytes to promote insulin secretion and fatty acid uptake respectively. In humans, levels of GIP positively correlate with obesity, and multiple genome-wide association studies have identified GIPR as a contributor to obesity. In addition, mice that lack GIPR or GIP

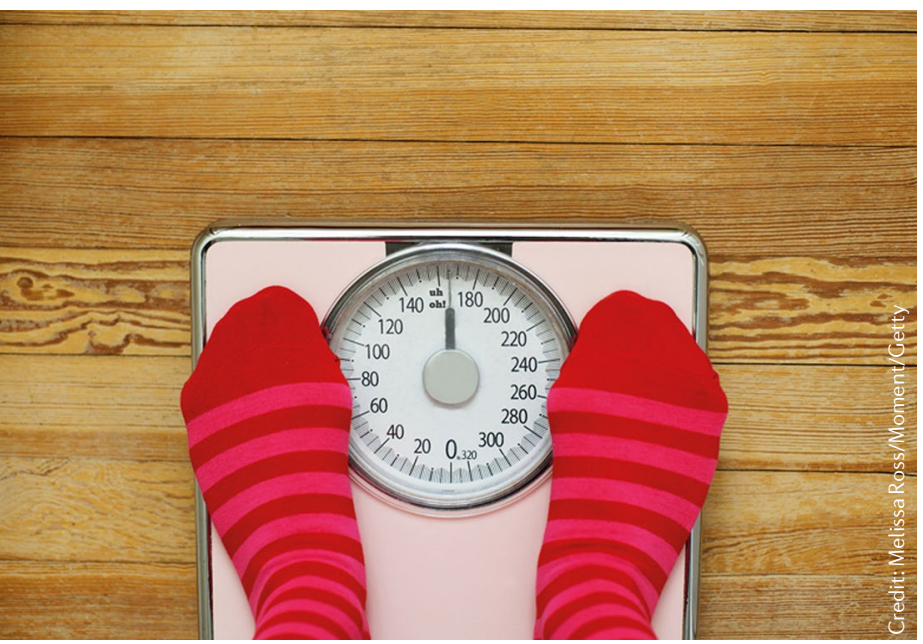

are resistant to diet-induced obesity (DIO). Given these findings, Lloyd and colleagues set out to develop therapeutic GIPR antagonists for the potential treatment of obesity.

First, the authors developed a mouse anti-GIPR antibody (muGIPR-Ab). In DIO mice fed a high-fat diet (HFD) for 11 weeks, intraperitoneal treatment with muGIPR-Ab for 45 days lowered fat mass by an average of $37 \%$ and reduced body weight. In addition, the treatment decreased fasting blood glucose and insulin concentrations without affecting glucose tolerance, decreased liver triglyceride concentrations and reduced food consumption and the respiratory exchange ratio.

Next, the authors compared their muGIPR-Ab with a current anti-obesity therapy, the GLP1 receptor (GLP1R) agonist liraglutide. In DIO mice fed a HFD for 30 weeks and treated for 38 days, muGIPR-Ab alone prevented body weight gain while liraglutide alone resulted in a $7.2 \%$ weight loss. Strikingly, the combination of muGIPR-Ab and liraglutide led to a $23.5 \%$ decrease in weight. Both liraglutide alone and the combination treatment reduced food intake by greater than $50 \%$ and improved glucose tolerance.

The enhanced weight loss achieved with the muGIPR-Ab and liraglutide combination also extended to the other GLP1R agonists exendin-4 and dulaglutide. Furthermore, muGIPR-Ab enhanced pre-established weight loss in DIO mice that had been treated with liraglutide for 2 weeks. Using GIPR $\beta$-cell-specific knockout mice, the beneficial effects of muGIPR-Ab and GLP1R agonism on body weight were shown to be independent of pancreatic $\beta$-cell GIPR.

Next, Lloyd and colleagues identified a human monoclonal anti-GIPR antibody (hGIPR-Ab) that selectively inhibited GIPR activity in cells expressing human GIPR by preventing GIP binding. The antibody similarly inhibited GIPR activity in primary human pancreatic microtissues and primary human subcutaneous adipocytes. Crystallographic studies confirmed that GIPR antagonism by hGIPR-Ab occurred via GIP displacement.

Finally, the authors further assessed the translational potential of their antibody using spontaneously obese male non-human primates (NHPs). Animals were treated for 18 days with either vehicle or dulaglutide, followed by treatment with or without hGIPR-Ab for an additional 30 days. As observed in mice, the combination treatment induced the greatest body weight reduction $(-14.5 \%)$ when compared with either hGIPR-Ab $(-1.86 \%)$ or dulaglutide $(-8.57 \%)$ alone, while vehicle-dosed NHPs gained $6.2 \%$ body weight during the study. Similarly, food intake was reduced in all treatment groups, with the greatest reduction observed in the combination group.

In summary, these findings demonstrate the therapeutic potential of intervening in the GIP-GIPR axis for the treatment of obesity. Further work is necessary to better understand the precise mechanism of action of anti-GIPR antibodies.

Sarah Crunkhorn

ORIGINAL ARTICLE Killion, E. A. et al. Antiobesity effects of GIPR antagonists alone and in combination with GLP-1R agonists in preclinical models. Sci. Transl Med. 10, eaat3392 (2018) 